\title{
Collective Specialization in Multi-Rover Systems
}

\author{
G. S. Nitschke, M. C. Schut, A. E. Eiben \\ Department of Computer Science \\ Vrije Universiteit, Amsterdam \\ De Boelelaan 1081a, 1081HV Amsterdam, The Netherlands \\ nitschke@cs.vu.nl, schut@cs.vu.nl,gusz@cs.vu.nl
}

\begin{abstract}
Categories and Subject Descriptors: I.2.11 Distributed Artificial Intelligence: Intelligent agents
\end{abstract}

General Terms: Experimentation, Algorithms, Design.

Keywords: Artificial life, Neural networks.

\begin{abstract}
Neuro-Evolution (NE) methods have been successfully applied to the rover task domain [1], [2]. However, extending this domain to include the notion of using NE to facilitate emergent specialization, in order to increase task performance, has not yet been investigated. We introduce the Collective Neuro Evolution (CONE) method, and compares its efficacy for designing specialization, with a conventional NE method. CONE and conventional NE were applied to an extension of the multi-rover task [1], [2] for the purpose of designing collective behavior. This task requires solutions for controlling groups of simulated autonomous vehicles (rovers) that seek to maximize the number of points of interest discovered in an unexplored environment (global evaluation function). Rovers operated in a discrete simulation environment, and used complementary sensors and actuators so as to maximize the global evaluation function.

Specialization was defined at both the individual and group level according to the frequency with which rover sensors and actuators were activated. An individual rover was defined as being specialized if a given action was executed for the majority $(\geq 50 \%)$ of a rovers lifetime. Likewise, a rover group was defined as being specialized if rovers with a given individual specialization constituted the majority $(\geq 50 \%)$ of rovers in a group. Emergent specialization, facilitated by the NE methods was measured at both the individual and rover group level. At any simulation time step, a rover could execute one action, which equated to using one of two sensor types (detection or evaluation), or one of two actuator types (movement or communication). If a rover spent the majority of its lifetime executing a single action, it would be labeled as an evaluator, detector, communicator, or mover. Likewise, rover groups were defined as evaluator, detector, communicator, or mover groups if a majority of the group consisted of rovers with a given individual specialization. A rover domain performance benchmark was defined via specifying individual rover specialization a priori. The REVAC parameter calibration method [3] used to evolve a
\end{abstract}

non-specialized rover group with a high task performance.

One contribution was the provision of the CONE method, which facilitated emergent specialization, at both the individual agent and group level, so as to increase group fitness in collective behavior tasks. Previous research elucidated that behavioral specialization, at both the individual and group level, is beneficial for task performance [4]. Here, the research hypothesis was that CONE is appropriate for deriving specialization at both the individual (rover controller), and at the group (composition of specialized rover controllers), where such specialization results in a high group fitness (performance). To test this hypothesis, the task performance and emergent specialization observed using the CONE method was compared to that of the conventional NE method, as well as a non-adaptive heuristic controller method. For both NE methods, each rover maintained and evolved its own population of genotypes. CONE evolved populations of neurons, from which complete neural network controllers were constructed, where as, the conventional NE method evolved populations of complete controllers.

Results indicate that the CONE method was appropriate for facilitating specialization at both the individual and group levels, where as, the conventional NE method only facilitated individual specialization. An inferior performance was exhibited by rover groups controlled by the conventional NE method, and by non-specialized rover groups using heuristic controllers. In both cases, this was theorized to be a consequence of a lack of group level specialization.

\section{REFERENCES}

[1] A. Agogino and K. Tumer. Efficient Evaluation Functions for Multi-Rover Systems. In, Proceedings of the Genetic and Evolutionary Computation Conference, pages: 1-12, Springer-Verlag, New York, USA, 2004.

[2] K. Tumer and A. Agogino. Coordinating Multi-Rover Systems: Evaluation Functions for Dynamic and Noisy Environments. In, Proceedings of the 2005 conference on Genetic and evolutionary computation, pages: 591-598, ACM Press, Washington DC, USA, 2005.

[3] V. Nannen and A. Eiben. A Method for Parameter Calibration and Relevance Estimation in Evolutionary Algorithms. In, Proceedings of the Genetic and Evolutionary Computation Conference, pages: 183-190, ACM Press, Seattle, USA, 2006.

[4] A. Eiben and G. Nitschke and M. Schut. Collective Specialization for Evolutionary Design of a Multi-Robot System. In Proceedings of the Second International Workshop on Swarm Robotics, pages: 197-213, Springer, Rome, Italy, 2006. 